
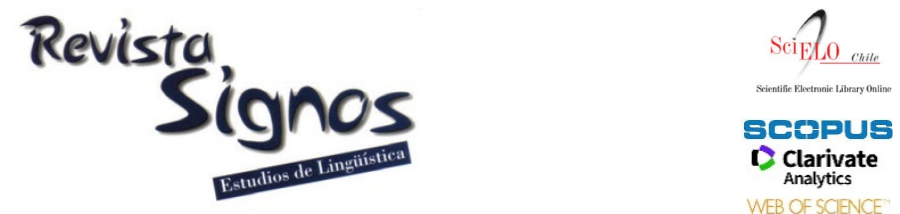

WEB OF SCIENCE

\title{
Potencial de Estructura Genérica en tesis de ingeniería eléctrica: Contrastes entre lenguas y niveles educativos*
}

\section{Generic Structure Potential in electric engineering dissertations: Contrasts between languages and educational stages}

\author{
Federico Navarro \\ UNIVERSIDAD DE O'HIGGINS \\ CHILE \\ federico.navarro@uoh.cl
}

\author{
Alex Caldas Simões \\ INSTITUTO FEDERAL DO ESPírito SANTO \\ BRASIL \\ alex.simoes@ifes.edu.br
}

Recibido: 02-VI-2018 / Aceptado: 28-VIII-2018

DOI: $10.4067 /$ S0718-09342019000200306

\section{Resumen}

Esta investigación describe la tesis en ingeniería eléctrica contrastando el nivel educativo y la lengua en dos universidades estatales metropolitanas tradicionales y altamente selectivas de Chile y Brasil. Desde un marco sistémico-funcional, se analizó cualitativamente la Configuración Contextual, la extensión, el Potencial de Estructura Genérica de elementos iniciales y de elementos semánticos y las opciones de despersonalización en la introducción y conclusión de 20 tesis recientes. La tesis se inscribe en un contexto de asimetría de experticia y saber entre autor y director, roles predominantemente masculinos, y varía en su complejidad y aporte según el nivel educativo. Consiste en una investigación individual sistemática sobre un tema especializado, en un marco de educación formal superior, y tiene como producto un texto escrito multimodal. Su extensión ronda las 96 páginas en pregrado y el doble de extensión en posgrado. Se inicia obligatoriamente con tapa, dedicatoria, agradecimientos y resumen en lengua local. El contraste entre lenguas muestra que en portugués se utilizan más elementos obligatorios relacionados con la protocolarización e internacionalización de ejemplares multimodales. Las introducciones incluyen obligatoriamente la motivación, objetivos y organización textual, mientras que en las conclusiones son obligatorias las consideraciones finales y trabajos futuros, y optativa la síntesis de hallazgos. En ambas secciones, la estructura es más compleja y estable en posgrado. La presencia del autor es despersonalizada casi por completo mediante estrategias como la metonimia. Esta investigación hace aportes al conocimiento del género y orienta iniciativas de alfabetización disciplinar en ingeniería de pregrado y posgrado en Chile y Brasil. 
Palabras Clave: Potencial de Estructura Genérica, Lingüística Sistémico-Funcional, escritura académica, géneros discursivos de formación, ingeniería eléctrica.

\begin{abstract}
This article describes electric engineering dissertations contrasting languages and educational stages in two metropolitan, traditional and highly selective state universities in Chile and Brazil. Drawing from Systemic-Functional Linguistics, the Contextual Configuration, extension, Generic Structure Potential of initial elements and of semantic elements and impersonalization options in introductions and conclusions of 20 recent dissertations were analyzed. Results show that dissertations take place in a knowledge and expertise asymmetrical relationship between mostly male writers and supervisors, and they vary in complexity and contribution due to educational stages. It consists of an individual systematic research on a specialized topic within a formal higher education setting and produces a multimodal written text. It is around 96-page long at the undergraduate level, but it doubles its length at the postgraduate level. It includes cover, dedication, acknowledgments and abstract in the local language as initial elements. Dissertations in Portuguese exhibit more compulsory elements related to protocolization and internationalization of multimodal texts than in dissertations in Spanish. Introductions must include motivation, goals and textual organization, whereas conclusions show compulsory final considerations and future studies, together with optional main findings; both sections are more complex in the postgraduate level. The author is impersonalized almost completely by means of strategies such as metonymy. This research helps understand the genre and provides insights to enhance literacy teaching in engineering within undergraduate and postgraduate programs in Chile and Brazil.
\end{abstract}

Key Words: Generic Structure Potential, Systemic-Functional Linguistics, Academic Writing, Students' genres, Electric Engineering.

\title{
INTRODUCCIÓN
}

En el contexto de la expansión del sistema de educación superior en Latinoamérica (Chiroleu \& Marquina, 2017), en la última década se ha acentuado el interés por los géneros académicos de formación que los estudiantes universitarios deben escribir a lo largo de las diferentes etapas educativas y contextos disciplinares. Las investigaciones han comenzado a abandonar la premisa de que los estudiantes muestran carencias formativas que redundan en textos deficitarios (Carlino, 2013), al tiempo que los escritos estudiantiles se han validado como el producto de prácticas legítimas, específicas, complejas, situadas, con propósitos sociales, educativos y comunicativos distintos a los de los escritos en otros niveles educativos o con otros niveles de experticia (Navarro, 2018). Los géneros académicos estudiantiles (Gardner \& Nesi, 2013), epistémicos (Thaiss \& Zawacki, 2006) o de formación buscan la enseñanza, aprendizaje y acreditación del conocimiento de los miembros novatos que ingresan a las culturas disciplinares en los espacios institucionalizados de las carreras en educación superior, y esta orientación institucional pedagógica y evaluativa redunda en rasgos sociales y discursivos particulares y diferenciados. 
Un género académico de formación que ha atraído especialmente la atención de los investigadores en Latinoamérica es la tesis. Se trata de una clase textual tipificada que tiene como objetivo general la construcción sistemática e individual de nuevo conocimiento relevante sobre una temática específica con base en bibliografía especializada y supervisión experta y, a lo largo de ese proceso escritural e investigativo, la formación de capital humano altamente calificado, un requisito central en la sociedad del conocimiento intensivo (Paré, Starke-Meyerring \& McAlpine, 2011). El género atraviesa distintos niveles educativos (pregrado, magíster, doctorado), existe en la mayoría de las carreras e instituciones, y por su complejidad discursiva constituye un desafío para la finalización de los estudios superiores, tanto de pregrado como posgrado (Caffarella \& Barnett, 2000). Esta complejidad no solo está dada por su extensión y por la demanda investigativa y formativa que implica, en ocasiones novedosa para el estudiante, sino también por su carácter híbrido, tanto en términos discursivos como contextuales: el escritor todavía no es un miembro autorizado experto en la comunidad disciplinar, y será evaluado por miembros con asimetría de poder, pero al mismo tiempo debe emular las prácticas letradas y epistemológicas de la comunidad experta y hacer aportes originales que modifiquen en cierta medida el saber consensuado (Russell, 2013). Así, es frecuente hallar estudiantes que han completado los cursos formativos obligatorios, pero que no logran o retrasan su titulación por la dificultad de finalizar su escrito de graduación (Swales, 2004).

Entre las investigaciones existentes en Latinoamérica, Arnoux (2009) ofrece los resultados de un proyecto colectivo en el que la escritura en posgrado se aborda desde múltiples perspectivas complementarias: la configuración socio-demográfica; las experiencias y representaciones de los escritores y su vínculo con los directores; los procesos de escritura de monografías, de apropiación de fuentes y reescritura en proyectos de tesis y de defensa oral; y la configuración discursiva y léxicogramatical del género. En un enfoque afín, Savio (2015) rastrea elementos discursivos disciplinarmente específicos (informalidad, valoración, opacidad, argumentación en espiral y subjetividad) en 8 tesis de maestría y doctorales de psicoanálisis de una universidad pública tradicional argentina. Pereira (2012) analiza, desde la teoría de la enunciación francesa y mediante procedimientos etnográficos y cualitativos (entrevistas a profesores y estudiantes), la producción de la tesis de pregrado o monografia en el área de Letras en una universidad de Brasil. Desde el mismo marco teórico, Medeiros (2015) da cuenta cualitativamente de la estructura de 6 resúmenes de tesis de maestría y doctorado de una universidad brasileña en términos de su categoría secuencial (descriptiva, narrativa, etc.) y su categoría informacional (tema, metodología, conclusión, etc.). Araújo (2006) aborda la estructura retórica, desde el marco del análisis de géneros, y la construcción autoral en la sección conclusiones de 10 tesis de doctorado del área de análisis del discurso escritas en inglés y portugués (defendidas en distintas universidades de Inglaterra y Brasil por hablantes nativos). Desde un marco teórico similar, Solar (2013) estudia 34 tesis doctorales de una 
universidad privada tradicional chilena para determinar la estructura retórica de movidas y pasos (convencionales y opcionales) de las introducciones, en este caso del área de química y lingüística. Martínez Hincapié (2015) también ofrece un contraste entre disciplinas de humanidades (Historia) y ciencias (Física) en su estudio de movidas y macromovidas retóricas en 92 tesis doctorales de España y Chile. Tapia Ladino y Burdiles (2012) siguen el mismo enfoque, pero en el nivel de pregrado, y codifican el marco referencial de 30 tesis de la carrera de Trabajo Social de una universidad privada tradicional chilena para delimitar movidas y pasos retóricos. Castro Azuara y Sánchez Camargo (2015) estudian 64 tesinas de las carreras de Lenguas modernas, Filosofía, Historia y Literatura hispanoamericana de una universidad estatal mexicana para determinar las variaciones disciplinares de su estructura retórica. Venegas, Núñez, Zamora y Santana (2015) ofrecen un trabajo colectivo que aborda 400 tesis de pregrado en distintas carreras de una universidad privada tradicional chilena y siguiendo una metodología mixta cualita-cuantitativa que da cuenta de múltiples dimensiones lingüísticas, abarcando tanto la estructura retórica como aspectos léxico-gramaticales. De forma similar, pero incluyendo también niveles de maestría y doctorado, Meza y Sabaj (2016) identifican la distribución de diferentes funciones discursivas de disenso y consenso en 36 tesis de universidades chilenas e interpretan los resultados a partir de su carácter de texto de transición en el ingreso a la comunidad experta.

Este recorrido por algunas investigaciones latinoamericanas recientes sobre géneros discursivos de formación no pretende exhaustividad, sino demostrar la producción sostenida, sistemática y agregable de conocimiento en la temática en un contexto de expansión del sistema de educación superior y de necesidad de apoyo en los procesos de aprendizaje y de alfabetización académica de los estudiantes, en particular en géneros de gran complejidad como la tesis. La mayoría de estas investigaciones están vinculadas directa o indirectamente a la promoción de iniciativas y la producción de instrumentos que promuevan la enseñanza de los géneros investigados. Tienen como rasgo común el interés por la investigación situada y ecológica de géneros discursivos de formación en tanto clases tipificadas de textos legítimos que se elaboran con propósitos educativos y que presentan variaciones según el área, disciplina, carrera e institución.

Son todavía escasas, sin embargo, las investigaciones sobre géneros académicos de formación que contrasten instituciones, idiomas y niveles educativos diferentes en América Latina, manteniendo como constante el género y la disciplina, a pesar de que los estudiantes de la región enfrentan desafíos en muchos casos similares, continúan escribiendo géneros de formación en distintos niveles formativos y se desplazan entre distintas universidades y países para completar sus estudios superiores. Un antecedente en esta línea es Navarro (2015), quien contrasta la configuración de la 
estructura retórica y las dinámicas de circulación del plan de negocios en portugués (Brasil) y español (Argentina) y en el ámbito estudiantil y experto.

Para llenar esta laguna, la presente investigación se propone describir la estructura de la tesis en ingeniería eléctrica contrastando el nivel educativo (pregrado y doctorado) y la lengua (español y portugués) en dos universidades estatales metropolitanas tradicionales y altamente selectivas de Chile y Brasil. Para tal fin, se codificará la configuración de la estructura semántica en introducciones y conclusiones de un corpus de 20 tesis recientes aprobadas, con asistencia del software de análisis cualitativo QSR NVivo 11 y posterior cuantificación de resultados. Estas secciones, delimitadas a priori de forma explícita por los autores, son fuertemente retóricas, porque suelen presentar el tema y argumentar la relevancia del problema, y por tanto pueden ser un importante desafío para el escritor en formación (Swales, 2004). En el caso de las introducciones a tesis, se espera que incluyan complejos recursos textuales de indicación de propósitos, hipótesis y preguntas de investigación (Lim, 2014). Además, estas secciones son especialmente sensibles a variaciones disciplinares (Feak \& Swales, 2011).

De forma complementaria, se describirá la Configuración Contextual del género, se identificarán los elementos textuales complementarios iniciales (dedicatoria, agradecimiento, epígrafe, resumen, etc.), se cuantificará la extensión de los ejemplares y se determinará la distribución de opciones léxico-gramaticales de despersonalización (García Negroni, 2008) utilizadas para reemplazar la primera persona gramatical singular coincidente. En suma, se dará cuenta de diferentes rasgos textuales y contextuales del género útiles para su caracterización y para su enseñanza en iniciativas de alfabetización disciplinar en educación superior, contrastando niveles educativos, lenguas y contextos institucionales.

Se utilizará la noción de Potencial de Estructura Genérica (Generic Structure Potential), postulada originalmente por Ruqaiya Hasan para abordar géneros como la prestación de servicios (economic encounter; Hasan, 1989) y el cuento infantil (nursery tale; Hasan, 1996), y que se ha empleado desde entonces para dar cuenta de la configuración semántico-estructural de géneros discursivos como los anuncios impresos (Yuen, 2004), la comunicación en call-centers (Xu, Wang, Forey \& Li, 2010), las propagandas impresas (Oyebode \& Unuabonah, 2013), y las sentencias penales (Agüero, 2014), entre otros (más detalles en Simões, 2016). Su utilidad para dar cuenta de géneros discursivos de estructura compleja que combinan modos verbales y visuales, como en el caso de la tesis en ingeniería eléctrica, ha sido postulada recientemente por Bowcher y Yameng Liang (2016).

\section{Potencial de estructura genérica}

Siguiendo los postulados fundamentales de la Lingüística Sistémico-Funcional (Halliday \& Matthiessen, 2014), Hasan propone que la cultura se realiza como "an 
integrated body of the total set of meanings available to a community" (Hasan, 1989: 99). Se trata de un 'Potencial Semiótico' que incluye formas de hacer, formas de ser y formas de decir en sociedad (Hasan, 1989). Este Potencial Semiótico se especifica a través del más importante de los sistemas semióticos existentes: el lenguaje. De esta manera, el 'Potencial Semántico' incluye el conjunto de significados conformados y expresados por el lenguaje. Este conjunto de significados se especifica en un tercer nivel de especificidad: el 'Potencial Semántico específico de un género discursivo', incluido el 'Potencial de Estructura Genérica'.

A su vez, la cultura se especifica en niveles consecutivos de especificidad contextual. Primero, la cultura se realiza en los valores situacionales significativos que establecen relaciones con el potencial semiótico de una cultura. Segundo, estos valores se especifican en el conjunto de los valores posibles de Campo, Tenor y Modo, es decir, el contexto de situación relevante para el lenguaje, ya que establecen relaciones bidireccionales con este. Por último, el conjunto de valores que realizan las variables de Campo, Tenor y Modo que resultan significativos en una cierta actividad social conforman la 'Configuración Contextual'. No se trata de la configuración de una situación específica, concreta e irrepetible, sino de un ‘tipo de situación’ dentro de las opciones que engloba una cultura. Hasan enfatiza la importancia de entender los valores situaciones combinados al explicar la aparición o ausencia de ciertos elementos textuales. En la Figura 1 a continuación, se sintetizan estas nociones clave y sus relaciones.

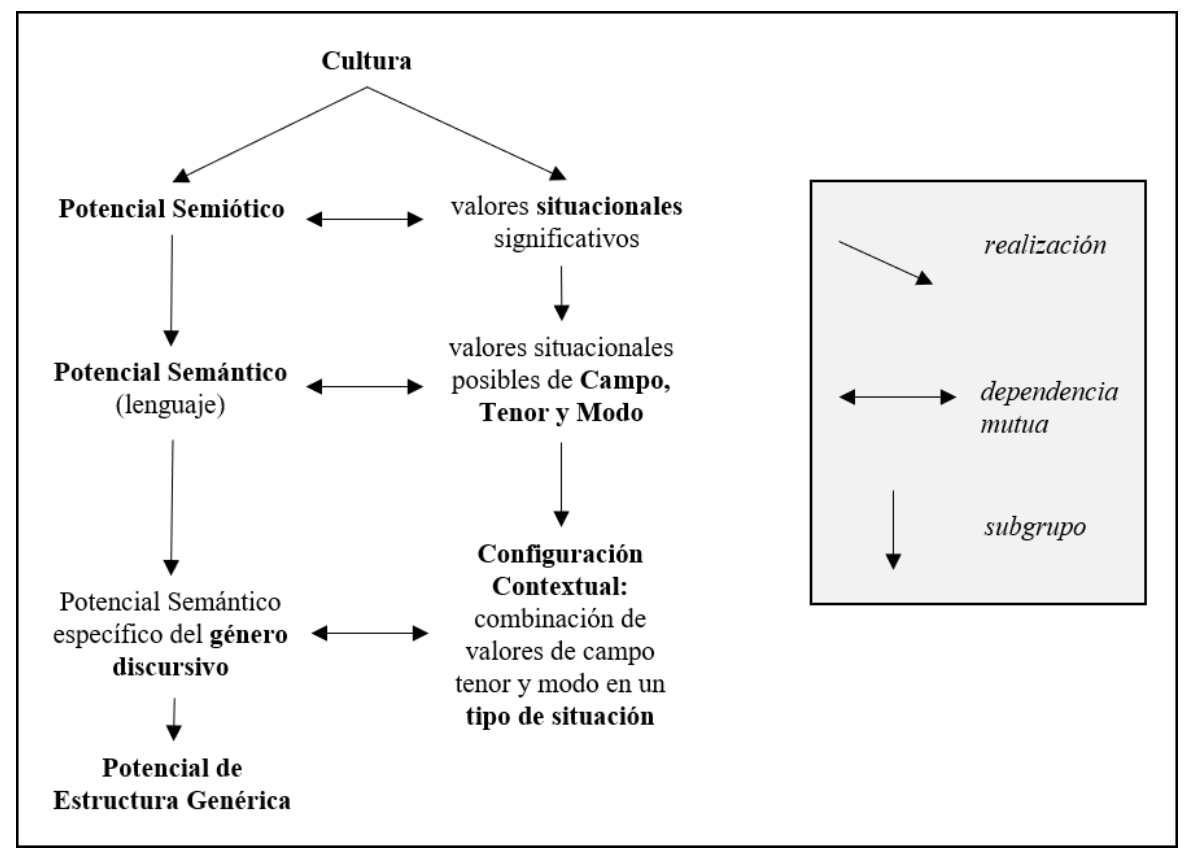

Figura 1. Potencial Semiótico, Potencial Semántico y Potencial de Estructura Genérica. Fuente: elaboración propia a partir de Hasan (1989). 
El 'género discursivo' se entiende como la manifestación verbal de la Configuración Contextual. En palabras de Hasan,

"if Contextual Configuration is a class of situation type, then genre is language doing the job appropriate to that class of social happenings" (Hasan, 1989: 108).

Por este motivo, a partir del contexto es posible anticipar los elementos textuales, al mismo tiempo que es posible reconstruir el contexto a partir de los elementos textuales, ya que ambas dimensiones establecen dependencias bidireccionales. En concreto, el 'Potencial de Estructura Genérica' engloba el conjunto de significados relevantes para la estructuración global de los textos, es decir, elementos que funcionan como etapas o stages con alguna consecuencia en la progresión textual (Hasan, 1989). Para abordar la estructura textual, Hasan distingue entre los elementos obligatorios, que deben realizarse textualmente y que definen el género discursivo del que se trate, y los elementos opcionales, responsables por las variaciones estructurales dentro de un mismo género y vinculados a variaciones en la Configuración Contextual. Estos elementos se organizan en una secuencia, o relación lineal. Por último, la autora postula la existencia de elementos iterativos, es (o decir, elementos obligatorios u opcionales que se realizan más de una vez a lo largo del texto con la función de expresar significados particulares. De esta manera, el Potencial de Estructura Genérica abarca la totalidad de significados textuales estructurales y se compone de los elementos obligatorios, opcionales e iterativos, organizados secuencialmente, que engloban todas las posibilidades estructurales para un texto en una determinada Configuración Contextual. Es importante destacar que la noción de potencial coloca el foco en el rango de posibles variaciones y opciones disponibles dentro de un mismo tipo o categoría. El texto será entendido como el resultado de un proceso de elecciones semánticas y lingüísticas y, por tanto, como un potencial de significado realizado. De esta forma, cada texto tiene una estructura particular diferente, pero cada una realiza una opción dentro de una estructura potencial genérica dada. El texto presenta entonces una 'unidad de estructura', construida mediante categorías semánticas ordenadas en una estructura, pero también una 'unidad de textura', construida mediante categorías léxico-gramaticales que contribuyen con la cohesión.

La noción de Potencial de Estructura Genérica constituye un poderoso instrumento teórico-metodológico para la investigación y la enseñanza explícita de las clases tipificadas de textos complejos: permite dar cuenta de una configuración jerárquica y secuencial de una estructura textual, incluyendo elementos iterativos; distingue entre diferentes grados de obligatoriedad, permitiendo distinguir entre textos de una misma clase y géneros discursivos distintos; vincula sistemáticamente las clases de textos a los tipos de contextos en los que cumplen roles situados significativos; incluye las variaciones de instanciación vinculadas a las opciones de los usuarios, sin poner en riesgo la tipificación como clase particular de texto; y permite relacionar 
sistemáticamente elementos estructurales y elementos léxicos y gramaticales. Para la descripción del Potencial de Estructura Genérica, Hasan propone una serie de signos gráficos, presentados a continuación (Hasan, 1989; Eggins, 2004):

\begin{tabular}{|c|c|}
\hline$\wedge$ secuencia & - más de una opción secuencial \\
\hline ( ) elemento opcional & [] restricción de opcionalidad secuencial \\
\hline$\hookleftarrow$ elemento iterativo & lementos con igual posibilidad de iteración \\
\hline
\end{tabular}

Figura 2. Signos para describir el Potencial de Estructura Genérica. Fuente: elaboración propia a partir de Hasan (1989).

\section{Corpus y metodología}

Para la construcción y análisis del corpus, se determinó como constante de análisis el área de ingeniería eléctrica. Dentro del ámbito complejo y diverso de las carreras vinculadas a la ingeniería y la tecnología, la ingeniería eléctrica se destaca por su centralidad, demostrada en la existencia de asociaciones internacionales de gran peso como la IEEE (Institution of Electrical and Electronics Engineers). Además, la carrera de ingeniería eléctrica es frecuente en el currículum universitario, a nivel regional e internacional, y ofrece titulaciones en diferentes niveles educativos, lo cual facilita el tertium comparationis o espacio común de comparación (Connor \& Moreno, 2005). En términos generales, se trata de un espacio disciplinar con carreras de pregrado y programas de posgrado y caracterizado por la elaboración de textos multimodales que combinan opciones léxico-gramaticales, visuales y matemáticas.

Se determinaron tres variables de contraste: institución (universidad estatal metropolitana tradicional y altamente selectiva de Chile o Brasil), lengua (español o portugués) y nivel educativo (pregrado o doctorado). A partir del banco de tesis digitales de cada institución, se seleccionaron al azar 20 textos recientes (menos de 5 años), según la siguiente configuración:

Tabla 1. Configuración de corpus de análisis.

\begin{tabular}{|c|c|c|}
\hline Ejemplares & Institución / Lengua & Nivel educativo \\
\hline 5 & \multirow{2}{*}{ brasileña / portugués } & pregrado \\
\cline { 3 - 3 } & & posgrado \\
\hline 5 & \multirow{2}{*}{ chilena / español } & pregrado \\
\cline { 3 - 3 } & & posgrado \\
\hline 5 &
\end{tabular}

Con la asistencia del software de análisis cualitativo QSR NVivo 11 y posterior cuantificación de resultados, se realizó una codificación de la estructura semántica potencial en las secciones explícitamente delimitadas de introducción y conclusión del corpus. Primero, el equipo de analistas identificó categorías emergentes tanto para los 
elementos textuales iniciales (detallados en la sección 3.3) como para los elementos que configuran la estructura de la introducción (ver sección 3.4) y de la conclusión (ver sección 3.5). Se elaboraron definiciones para cada elemento y se identificaron ejemplos típicos de realización. Luego de este proceso, se construyó un árbol de nodos dentro de NVivo, con las categorías y subcategorías de la estructura semántica potencial de las secciones; sobre el corpus digitalizado, se codificó cada fragmento textual según su pertenencia a los distintos nodos. Dadas las dificultades para garantizar la consistencia cualitativa en la identificación de categorías retóricas estructurales (Swales, 2004), se optó por la metodología de codificación conjunta (Eodice, Geller \& Lerner, 2016); de esta manera, se fomentó la discusión permanente dentro del equipo y los consensos de análisis sobre fragmentos del corpus.

Finalizada esta etapa, se obtuvo del software una base de datos con la cantidad de ocurrencias para cada nodo en relación a las variables del corpus (institución/lengua y nivel educativo). Esta base de datos permitió clasificar los elementos en términos de secuencia, grado de obligatoriedad y carácter iterativo de los componentes. Para los fines de esta investigación y considerando el número relativamente bajo de ocurrencias, se determina que un elemento obligatorio debe presentar una frecuencia igual o superior al $80 \%$ y un elemento optativo una frecuencia igual o superior al $40 \%$; los elementos con menor frecuencia serán considerados incidentales, sin evidencia suficiente como para considerarlos estables dentro del Potencial de Estructura Genérica. Investigaciones afines han fijado porcentajes de frecuencia de corte similares o menores para considerar a un elemento como obligatorio u optativo; Kanoksilapatham (2007), por ejemplo, establece un 60\% de ocurrencias para distinguir entre elementos obligatorios y optativos; investigaciones regionales también se han interesado por dar cuenta del porcentaje de ocurrencias de elementos estructurales en tanto criterio central para la identificación de unidades organizacionales de tipo retórico (Ibáñez, 2008; Parodi, 2008).

Por último, se codificó y cuantificó la distribución de opciones léxico-gramaticales de personalización (primera persona singular coincidente) y despersonalización utilizadas en introducciones y conclusiones. Para tal fin, se distinguió entre formas no finitas (uso de infinitivo para neutralizar el agente del proceso evocado); voz pasiva (pasivas con 'ser' y con 'se', que permiten dejar indeterminado a quién se considera responsable de la acción); metonimia (atribución al texto o a la investigación de las intenciones, tesis o conclusiones del autor); primera persona plural (nosotros genérico, referido a grupos sociales o la humanidad en general; nosotros inclusivo, en el que el lector queda incorporado en el mismo grupo del autor; y nosotros de modestia, en el que la referencia es el propio autor); y primera persona singular (persona coincidente para textos con un solo autor, como la tesis) (García Negroni, 2008).

De forma complementaria, se determinaron los rasgos relevantes de la Configuración Contextual del género, se identificaron los elementos textuales 
complementarios iniciales (dedicatoria, agradecimiento, epígrafe, resumen, etc.) y se cuantificó la extensión (media y mediana) en páginas y palabras de los ejemplares.

\section{Resultados y discusión}

\subsection{Configuración Contextual}

La Configuración Contextual de los diferentes ejemplares del corpus es similar: el campo consiste en una investigación de un autor individual sobre un problema específico en un marco de educación formal superior, con frecuencia una investigación aplicada a resolver un problema real en la industria o a mejorar una técnica, proceso o material. El texto elaborado corresponde al producto final de un período de estudio e investigación extendido, en el que el estudiante-escritor tiene un rol más autónomo e independiente que en otras instancias formativas previas. El tenor muestra una relación de asimetría de poder y de saber entre el autor responsable y el director o directores, uno o dos profesores con formación académica superior al nivel de investigación emprendida, si bien suele haber cercanía social. Esta asimetría implica tanto la formación y acompañamiento del aprendizaje por parte del director, como también su evaluación continua. Una vez concluida la investigación, el texto debe ser enviado a un tribunal conformado por profesores del Departamento e invitados externos, que debe evaluarlo. El canal es gráfico y el medio es escrito, en articulación con otros modos semióticos (visual, lenguaje matemático). El lenguaje desempeña un rol constitutivo o central en el contexto y el proceso de intercambio es pasivo, ya que el lector no co-construye el texto con el autor.

Sin embargo, existen variaciones en la profundización de la investigación, la innovación de los aportes y el rol de experticia interpretado por el estudiante-escritor, además de la denominación de la clase de texto. La investigación de pregrado implica alrededor de 12 meses de duración y la finalidad es ahondar en el conocimiento de alguna temática, mediante procedimientos sistemáticos e informados teóricamente, que demuestren la formación previa. Se trata, por tanto, de la elaboración de un producto textual derivado de los cursos de pregrado. La investigación de posgrado, en cambio, requiere una investigación de 4-6 años de duración que presente aportes originales al conocimiento científico y tecnológico consensuado. Por lo tanto, la relevancia e innovación del problema planteado y los resultados hallados son mayores en profundidad y alcance. En este sentido, hay un contraste en la orientación y las dinámicas de formación, por un lado, y en el grado de cercanía con géneros y prácticas profesionales, por el otro, que permite distinguir la tesis de pregrado de la tesis de posgrado como dos configuraciones distintas de un mismo género de introducción a la investigación (Caffarella \& Barnett, 2000).

Las denominaciones varían: 'memoria' ('memoria para optar al título de ingeniero civil eléctrico') para la tesis de pregrado en español en la universidad chilena y 
'monografía' (monografial Trabalho de Conclusão de Curso (TCC)) para la tesis de pregrado en portugués en la universidad brasileña; en contraste, la denominación 'tesis' es común a ambas instituciones y lenguas para el nivel de posgrado (tese de doutorado/pósgraduação en portugués y 'tesis para optar al grado de doctor en ingeniería eléctrica' en español).

Otro elemento relevante de la Configuración Contextual en las ingenierías es la distribución de género, ya que las carreras suelen tener predominancia masculina: en la Universidad de Chile, por ejemplo, la matrícula de primer año de carreras tecnológicas muestra un 77\% de hombres (Bonilla Dávila, 2016). En Brasil, la situación es similar; por ejemplo, la matrícula de las Escuelas de Ingeniería de la Universidade de São Paulo presenta un $76 \%$ de estudiantes hombres en pregrado y un $72 \%$ en posgrado (Controladoria Geral, 2016). Estos datos contrastan con la distribución de estudiantes en educación superior en general, que muestran números más balanceados por género. Sin embargo, son consistentes con las investigaciones internacionales sobre las brechas de género en carreras vinculadas a ciencia, tecnología, ingeniería y matemáticas (STEM, por sus iniciales en inglés): solo el 20\% de los títulos de pregrado son obtenidos por mujeres, y esta participación se reduce aún más en los niveles de posgrado y de transición al ámbito laboral (Hill, Corbett \& Rose, 2010).

Al analizar el corpus por género de autores y directores se halla, de forma consistente con las tendencias mencionadas, una predominancia masculina de actores vinculados a la tesis de ingeniería eléctrica en todos los niveles educativos, contextos institucionales y lenguas, como se aprecia en la Tabla 2:

Tabla 2. Cantidad y porcentaje de hombres y mujeres como autores y directores de tesis de ingeniería eléctrica por nivel educativo y lengua/institución.

\begin{tabular}{|c|c|c|c|c|c|c|c|c|c|c|c|}
\hline & \multicolumn{2}{|c|}{$\begin{array}{c}\text { Pregrado } \\
\text { Portugués/Brasil }\end{array}$} & \multicolumn{2}{|c|}{$\begin{array}{c}\text { Pregrado } \\
\text { Español/Chile }\end{array}$} & \multicolumn{2}{|c|}{$\begin{array}{c}\text { Posgrado } \\
\text { Portugués/Brasil }\end{array}$} & \multicolumn{2}{|c|}{$\begin{array}{c}\text { Posgrado } \\
\text { Español/Chile }\end{array}$} & \multicolumn{2}{|c|}{ TOTAL } \\
\hline & & n. & $\%$ & n. & $\%$ & n. & $\%$ & n. & $\%$ & n. & $\%$ \\
\hline \multirow{2}{*}{ Autores } & Hombres & 4 & 80 & 5 & 100 & 3 & 60 & 3 & 60 & 15 & 75 \\
\hline & Mujeres & 1 & 20 & 0 & 0 & 2 & 40 & 2 & 40 & 5 & 25 \\
\hline \multirow{2}{*}{ Directores } & Hombres & 5 & 100 & 5 & 100 & 3 & 60 & 5 & 100 & 18 & 90 \\
\hline & Mujeres & 0 & 0 & 0 & 0 & 2 & 40 & 0 & 0 & 2 & 10 \\
\hline
\end{tabular}

Los resultados muestran una predominancia de hombres del 75\% entre los autores y del 90\% entre los directores, es decir, se trata de un género escrito en su mayor parte por hombres y cuyos orientadores y evaluadores con base institucional son casi exclusivamente hombres. Esta tendencia muestra su mayor predominancia en el contexto chileno ( $80 \%$ de autores y $100 \%$ de directores hombres), al tiempo que se balancea un poco en el posgrado de ambos contextos institucionales, donde la distribución de autores es $60 \%$ hombres. Este relativo balance de género en posgrado parece diferenciarse de la tendencia inversa hallada por la bibliografía (Hill et al., 2010), si bien el número reducido de ejemplares no permite llegar a conclusiones definitivas. 


\subsection{Extensión de tesis}

A continuación, se determina la extensión en páginas y palabras de los ejemplares del corpus, contrastando niveles educativos y lenguas; se busca identificar, además, el rango de variación habilitado. La Tabla 3 expone los resultados hallados:

Tabla 3. Media y mediana de extensión en páginas y palabras de tesis de ingeniería eléctrica por nivel educativo y lengua/institución.

\begin{tabular}{|c|c|c|c|c|c|c|c|c|c|c|c|c|}
\hline & \multirow{2}{*}{\multicolumn{2}{|c|}{\begin{tabular}{|c|} 
Pregrado \\
Portugués/Brasil
\end{tabular}}} & \multirow{2}{*}{\multicolumn{2}{|c|}{$\begin{array}{c}\text { Pregrado } \\
\text { Español/Chile }\end{array}$}} & \multirow{2}{*}{\multicolumn{2}{|c|}{ Total pregrado }} & \multirow{2}{*}{\multicolumn{2}{|c|}{$\begin{array}{c}\text { Posgrado } \\
\text { Portugués/Brasil }\end{array}$}} & \multirow{2}{*}{\multicolumn{2}{|c|}{$\begin{array}{c}\text { Posgrado } \\
\text { Español/Chile }\end{array}$}} & \multirow{2}{*}{\multicolumn{2}{|c|}{ Total posgrado }} \\
\hline & & & & & & & & & & & & \\
\hline & Media & Mediana & Media & Mediana & Media & Mediana & Media & Mediana & Media & Mediana & Media & Mediana \\
\hline Páginas & 101 & 89 & 90 & 93 & 96 & 91 & 178 & 141 & 194 & 170 & 186 & 156 \\
\hline Palabras & 17648 & 14929 & 25076 & 25951 & 21362 & 20703 & 52759 & 42826 & 62680 & 59208 & 57720 & 45904 \\
\hline
\end{tabular}

Los datos muestran que las diferencias contextuales descritas antes (complejidad del aporte, extensión de la investigación, formación y experticia del autor) impactan en una extensión diferente según el nivel educativo: las tesis de pregrado tienen 96 páginas/21362 palabras en promedio (mediana: 91 páginas/20703 palabras), mientras que las tesis de posgrado promedian las 186 páginas/57720 palabras (mediana: 156 páginas/45904 palabras). Sin embargo, esta diferencia de extensión no parecería, en principio, tan marcada: las tesis doctorales presentan aproximadamente el doble de extensión que las tesis de pregrado, a pesar de los importantes contrastes del rol de los escritores. A su vez, las diferencias entre la media y la mediana sugieren que las tesis tienen una dispersión amplia de extensiones, sobre todo en el posgrado: las tesis doctorales brasileñas van de 107 a 349 páginas, mientras que las chilenas presentan un rango de variación de 103 a 386; por el contrario, la mayor homogeneidad de extensión está en el pregrado chileno, entre 75 y 100 páginas, lo que acerca la media y mediana de extensión en páginas y palabras. Por último, al comparar entre lenguas y contextos institucionales, se halla que las tesis chilenas en español de pregrado son más breves que las tesis brasileñas en español correspondientes, pero en el nivel de posgrado esta tendencia se invierte: las tesis de posgrado chilenas en español son más extensas que las brasileñas. Estos datos podrían estar indicando un mayor contraste pregrado/posgrado en demandas de producción textual, al menos en términos de extensión, en el contexto chileno.

\subsection{Potencial de estructura genérica de elementos textuales iniciales}

A continuación, se analiza la secuencia, iteratividad y grado de obligatoriedad de un conjunto de elementos que pueden potencialmente aparecer de forma previa al texto propiamente dicho de la tesis y contribuir a su reconocimiento como ejemplar del género: tapa, portada, ficha de catalogación, hoja de evaluación, dedicatoria, agradecimientos, epígrafe, resumen en lengua local, abstract en inglés, lista de figuras, lista de tablas, lista de símbolos y lista de siglas. La tapa carece de la indicación de 
director y programa de estudios, mientras que la portada sí ofrece esos datos. Por su parte, la ficha de catalogación incluye los datos para la incorporación de la tesis en la biblioteca, como autor, título, año, números de páginas, palabras clave, entre otros. Asimismo, la hoja de evaluación consiste en una breve evaluación del trabajo por parte del comité evaluador e incluye fecha, indicación de aprobación y una calificación. Por último, aunque vinculadas, la dedicatoria es mucho más breve que los agradecimientos. La Tabla 4 especifica la presencia relativa de estos elementos en el corpus de análisis y la Tabla 5 los potenciales de estructura genérica; en fondo negro aparecen las frecuencias de elementos considerados obligatorios $(\geq 80 \%)$, en fondo gris los elementos optativos $(\geq 40 \%)$ y en fondo blanco los elementos accidentales que, con las evidencias disponibles, no pueden postularse como parte del género discursivo.

Tabla 4. Cantidad y porcentaje de elementos estructurales iniciales en tesis de ingeniería eléctrica por nivel educativo y lengua/institución.

\begin{tabular}{|c|c|c|c|c|c|c|c|c|c|c|}
\hline & \multicolumn{2}{|c|}{$\begin{array}{c}\text { Pregrado } \\
\text { Portugués/Brasil } \\
\end{array}$} & \multicolumn{2}{|c|}{$\begin{array}{c}\text { Pregrado } \\
\text { Español/Chile }\end{array}$} & \multicolumn{2}{|c|}{$\begin{array}{c}\text { Posgrado } \\
\text { Portugués/Brasil }\end{array}$} & \multicolumn{2}{|c|}{$\begin{array}{c}\text { Posgrado } \\
\text { Español/Chile }\end{array}$} & \multicolumn{2}{|c|}{ TOTAL } \\
\hline & n. & $\%$ & n. & $\%$ & n. & $\%$ & n. & $\%$ & n. & $\%$ \\
\hline Tapa (TA) & 5 & 100 & 5 & 100 & 5 & 100 & 5 & 100 & 20 & 100 \\
\hline Portada (PO) & 4 & 80 & 0 & 0 & 5 & 100 & 0 & 0 & 9 & 45 \\
\hline Catalogación (CA) & 5 & 100 & 0 & 0 & 5 & 100 & 0 & 0 & 10 & 50 \\
\hline Evaluación (EV) & 5 & 100 & 0 & 0 & 0 & 0 & 0 & 0 & 5 & 25 \\
\hline Dedicatoria (DE) & 5 & 100 & 1 & 20 & 2 & 40 & 4 & 80 & 12 & 60 \\
\hline Agradecimientos (AG) & 5 & 100 & 4 & 80 & 5 & 100 & 5 & 100 & 19 & 95 \\
\hline Epígrafe (EP) & 2 & 40 & 2 & 40 & 3 & 60 & 1 & 20 & 8 & 40 \\
\hline Resumen (RE) & 5 & 100 & 5 & 100 & 5 & 100 & 5 & 100 & 20 & 100 \\
\hline Abstract (AB) & 5 & 100 & 0 & 0 & 5 & 100 & 2 & 40 & 12 & 60 \\
\hline L. Figuras (LF) & 5 & 100 & 2 & 40 & 5 & 100 & 2 & 40 & 14 & 70 \\
\hline L. Tablas (LT) & 4 & 80 & 2 & 40 & 5 & 100 & 2 & 40 & 13 & 65 \\
\hline L. Símbolos (LSB) & 1 & 20 & 0 & 0 & 1 & 20 & 0 & 0 & 2 & 10 \\
\hline L. Siglas (LSG) & 4 & 80 & 1 & 20 & 5 & 100 & 0 & 0 & 10 & 50 \\
\hline
\end{tabular}

Tabla 5. Potencial de estructura genérica de elementos iniciales a tesis de ingeniería eléctrica por nivel educativo y lengua/institución.

\begin{tabular}{|l|l|}
\hline Total tesis & $\mathrm{TA}^{\wedge}(\mathrm{PO})^{\wedge}(\mathrm{CA})^{\wedge} \mathrm{DE}^{\wedge} \mathrm{AG} \mathrm{G}^{\wedge}(\mathrm{EP})^{\wedge} \mathrm{RE}^{\wedge}(\mathrm{AB})^{\wedge}(\mathrm{LF})^{\wedge}(\mathrm{LT})^{\wedge}(\mathrm{LSG})$ \\
\hline Pregrado & $\mathrm{TA}^{\wedge}(\mathrm{PO})^{\wedge}(\mathrm{CA})^{\wedge} \mathrm{DE}^{\wedge} \mathrm{AG}{ }^{\wedge}(\mathrm{EP})^{\wedge} \mathrm{RE}^{\wedge}(\mathrm{AB})^{\wedge}(\mathrm{LF})^{\wedge}(\mathrm{LT})^{\wedge}(\mathrm{LSG})$ \\
\hline Posgrado & $\mathrm{TA}^{\wedge}(\mathrm{PO})^{\wedge}(\mathrm{CA})^{\wedge}(\mathrm{DE})^{\wedge} \mathrm{AG} \mathrm{G}^{\wedge}(\mathrm{EP})^{\wedge} \mathrm{RE}^{\wedge}(\mathrm{AB})^{\wedge}(\mathrm{LF})^{\wedge}(\mathrm{LT})^{\wedge}(\mathrm{LSG})$ \\
\hline Portugués/Brasil & $\mathrm{TA}^{\wedge} \mathrm{PO} \mathrm{PO}^{\wedge} \mathrm{CA}^{\wedge}(\mathrm{EV})^{\wedge}(\mathrm{DE})^{\wedge} \mathrm{AG}^{\wedge}(\mathrm{EP})^{\wedge} \mathrm{RE}^{\wedge} \mathrm{AB}^{\wedge} \mathrm{LF}^{\wedge} \mathrm{LT}^{\wedge} \mathrm{LSG}$ \\
\hline Español/Chile & $\mathrm{TA}^{\wedge}(\mathrm{DE})^{\wedge} \mathrm{AG}^{\wedge} \mathrm{RE}^{\wedge}(\mathrm{LF})^{\wedge}(\mathrm{LT})$ \\
\hline
\end{tabular}

Como puede observarse, el corpus total de tesis muestra que la secuencia jerárquica de elementos obligatorios previos al texto propiamente dicho incluye la tapa, dedicatoria, agradecimientos y resumen en la lengua local. Estos elementos son obligatorios en todos los subcorpus, salvo la dedicatoria en las tesis en español. Además, aparecen como elementos optativos portada, catalogación, epígrafe, abstract en inglés y listas de figuras, tablas y siglas. La comparación entre niveles educativos exhibe una llamativa continuidad, con los mismos elementos obligatorios y optativos, 
salvo la dedicatoria (solo obligatoria en pregrado). El mayor contraste aparece al comparar entre países y lenguas: las tesis brasileñas incluyen muchos más elementos obligatorios (portada, catalogación, abstract en inglés y listas de figuras, tablas y siglas) y optativos (evaluación, epígrafe) que las tesis chilenas.

Cabe destacar la llamativa ausencia del abstract en inglés en las tesis de pregrado y su bajo número en tesis de posgrado chilenas, un rasgo que puede atentar contra su catalogación, circulación e internacionalización. De la misma manera, las tesis brasileñas exhiben mayores rasgos de formato institucionalizado, con la presencia de portada, catalogación y evaluación (esta última, solo para el nivel de pregrado). Por otro lado, es llamativa la obligatoriedad de los agradecimientos, e incluso el número relativamente elevado de dedicatorias; se trata de elementos estructurales no estrictamente académicos en los que cobran relevancia aspectos vinculados al tenor, es decir, a los vínculos, los roles y las relaciones de cortesía con pares, directores y familiares y amigos del autor. Por último, es interesante constatar que en las tesis brasileñas la sistematización en forma de listas de los elementos no verbales (figuras, tablas, símbolos) tiene un peso mucho mayor que en las tesis chilenas, quizás indicando un carácter más multimodal.

\subsection{Potencial de estructura genérica de introducciones}

A continuación, se analiza la secuencia, iteratividad y grado de obligatoriedad de elementos estructurales de introducciones que aparecen en más de uno de los ejemplares del corpus completo: motivación, propuestas, hipótesis, estado del arte, fundamentación general, fundamentación teórica, alcances, objetivos, justificación, metodología y organización. Las propuestas explican de qué forma se pondrán en práctica las ideas del proyecto, mientras que los objetivos son una lista específica de verbos mostrando las acciones que se busca alcanzar con el trabajo. La fundamentación general es amplia y aborda aspectos relacionados con distintas dimensiones del trabajo, mientras que la fundamentación teórica presenta solamente las discusiones relativas a los conceptos y marcos conceptuales que informan la investigación. La Tabla 6 especifica la presencia relativa de estos elementos en el corpus de análisis y la Tabla 7 los potenciales de estructura genérica; se utiliza 4 para señalar los elementos iterativos: 
Tabla 6. Cantidad y porcentaje de elementos estructurales en introducciones de tesis de ingeniería eléctrica por nivel educativo y lengua/institución.

\begin{tabular}{|c|c|c|c|c|c|c|c|c|c|c|}
\hline & \multicolumn{2}{|c|}{$\begin{array}{c}\text { Pregrado } \\
\text { Portugués/Brasil } \\
\end{array}$} & \multicolumn{2}{|c|}{$\begin{array}{c}\text { Pregrado } \\
\text { Español/Chile }\end{array}$} & \multicolumn{2}{|c|}{$\begin{array}{c}\text { Posgrado } \\
\text { Portugués/Brasil } \\
\end{array}$} & \multicolumn{2}{|c|}{$\begin{array}{c}\text { Posgrado } \\
\text { Español/Chile }\end{array}$} & \multicolumn{2}{|c|}{ TOTAL } \\
\hline & $n$. & $\%$ & $n$. & $\%$ & $n$. & $\%$ & $n$. & $\%$ & $n$. & $\%$ \\
\hline Motivación MO & 3 & 60 & 4 & 80 & 5 & 100 & 4 & $80<$ & 16 & 80 \\
\hline Propuestas PR & 2 & 40 & 0 & 0 & 1 & 20 & 0 & 0 & 3 & 15 \\
\hline Hipótesis HI & 0 & 0 & 1 & 20 & 2 & 40 & 1 & 20 & 4 & 20 \\
\hline Estado del arte EA & 0 & 0 & 1 & 20 & 1 & 20 & 2 & $40<$ & 4 & 20 \\
\hline $\begin{array}{l}\text { Fundamentación general } \\
\text { FG }\end{array}$ & 3 & 60 & 0 & 0 & 2 & 40 & 2 & 40 & 7 & 35 \\
\hline $\begin{array}{l}\text { Fundamentación teórica } \\
\text { FT }\end{array}$ & 0 & 0 & 2 & $40<$ & 1 & 20 & 3 & 604 & 6 & 30 \\
\hline Alcances AL & 0 & 0 & 2 & 40 & 0 & 0 & 2 & 40 & 4 & 20 \\
\hline Objetivos OB & 5 & 100 & 5 & 100 & 5 & $100<$ & 5 & 1004 & 20 & 100 \\
\hline Justificación JU & 2 & 40 & 1 & 20 & 1 & 204 & 2 & 40 & 6 & 30 \\
\hline Metodología ME & 1 & 20 & 1 & 20 & 2 & $40<$ & 2 & 40 & 6 & 30 \\
\hline Organización OR & 3 & 60 & 5 & 100 & 4 & 80 & 5 & 100 & 17 & 85 \\
\hline
\end{tabular}

Tabla 7. Potencial de estructura genérica de introducción a tesis de ingeniería eléctrica por nivel educativo y lengua/institución.

\begin{tabular}{|l|l|}
\hline Total tesis & $\mathrm{MO}^{\wedge} \mathrm{OB}^{\wedge} \mathrm{OR}$ \\
\hline Pregrado & $(\mathrm{MO})^{\wedge} \mathrm{OB}^{\wedge} \mathrm{OR}$ \\
\hline Posgrado & $\mathrm{MO}^{\wedge}(\mathrm{FG})^{\wedge}(\mathrm{FT})^{\wedge} \mathrm{OB} \mathbf{\wedge}^{\wedge}(\mathrm{ME})^{\wedge} \mathrm{OR}$ \\
\hline Portugués/Brasil & $\mathrm{MO}^{\wedge}(\mathrm{FG})^{\wedge} \mathrm{OB}^{\wedge}(\mathrm{OR})$ \\
\hline Español/Chile & $\mathrm{MO}^{\wedge}(\mathrm{FT})^{\wedge}(\mathrm{AL})^{\wedge} \mathrm{OB}^{\wedge} \mathrm{OR}$ \\
\hline
\end{tabular}

A diferencia del Potencial de Estructura Genérica de elementos iniciales, las posibilidades estructurales en las introducciones muestran una gran dispersión. Por este motivo, los elementos obligatorios y optativos propios del género y disciplina son escasos: motivación y objetivos de la investigación, junto con su organización textual. Por el contrario, numerosos elementos son optativos solamente dentro de los subcorpus: las propuestas en la tesis de pregrado en portugués; las hipótesis en las tesis de posgrado en portugués; el estado del arte en las tesis de posgrado en español; y la justificación en las tesis de pregrado en español y de posgrado en portugués. $\mathrm{Al}$ focalizar en los niveles educativos, las tesis de posgrado muestran un Potencial de Estructura Genérica más complejo y estable que las tesis de pregrado: junto con los tres componentes obligatorios generales (motivación, objetivos iterativos y organización), aparecen como optativos la fundamentación general, la fundamentación teórica y la metodología, inexistentes en pregrado. Además, como se aprecia en la Tabla 6, aparecen numerosos elementos iterativos, asociados por tanto a la escritura con mayor grado de experticia. Al contrastar por lengua e institución, las tesis chilenas en español incorporan los objetivos como elemento obligatorio y los alcances y fundamentación teórica como elementos optativos; por su parte, las tesis brasileñas en portugués no muestran un potencial de estructura común, es decir, ofrecen más contrastes por niveles educativos. 


\subsection{Potencial de estructura genérica de conclusiones}

A continuación, se analiza la secuencia, iteratividad y grado de obligatoriedad de elementos de conclusiones que aparecen en más de uno de los ejemplares del corpus completo: consideraciones finales, contribuciones de la investigación, síntesis de lo investigado, dificultades enfrentadas durante la investigación, trabajos e investigaciones recomendadas para el futuro y publicaciones (las cuales consisten en la indicación de trabajos de la tesis que ya fueron publicados en revistas científicas o presentados en congresos). La Tabla 8 especifica la presencia relativa de estos elementos en el corpus de análisis y la Tabla 9 los potenciales de estructura genérica:

Tabla 8. Cantidad y porcentaje de elementos estructurales en conclusiones de tesis de ingeniería eléctrica por nivel educativo y lengua/institución.

\begin{tabular}{|c|c|c|c|c|c|c|c|c|c|c|}
\hline & \multicolumn{2}{|c|}{$\begin{array}{c}\text { Pregrado } \\
\text { Portugués/Brasil }\end{array}$} & \multicolumn{2}{|c|}{$\begin{array}{c}\text { Pregrado } \\
\text { Español/Chile }\end{array}$} & \multicolumn{2}{|c|}{$\begin{array}{c}\text { Posgrado } \\
\text { Portugués/Brasil }\end{array}$} & \multicolumn{2}{|c|}{$\begin{array}{c}\text { Posgrado } \\
\text { Español/Chile }\end{array}$} & \multicolumn{2}{|c|}{ TOTAL } \\
\hline & $n$. & $\%$ & $n$. & $\%$ & $n$. & $\%$ & $n$. & $\%$ & $n$. & $\%$ \\
\hline Consideraciones finales CF & 4 & 80 & 5 & 100 & 5 & 100 & 5 & 1004 & 19 & 95 \\
\hline Contribuciones $\mathrm{CO}$ & 1 & 20 & 0 & 0 & 1 & 20 & 5 & 100 & 7 & 35 \\
\hline Síntesis SI & 2 & 40 & 3 & 60 & 2 & 40 & 5 & 100 & 12 & 60 \\
\hline Dificultades DI & 1 & 20 & 0 & 0 & 1 & 20 & 0 & 0 & 2 & 10 \\
\hline Trabajos futuros TF & 5 & 100 & 5 & 100 & 3 & 60 & 4 & $80<$ & 17 & 85 \\
\hline Publicaciones PU & 2 & 40 & 0 & 0 & 0 & 0 & 0 & 0 & 2 & 10 \\
\hline
\end{tabular}

Tabla 9. Potencial de estructura genérica de conclusión a tesis de ingeniería eléctrica por nivel educativo y lengua/institución.

\begin{tabular}{|l|l|}
\hline Total tesis & $\mathrm{CF}^{\wedge}(\mathrm{SI})^{\wedge} \mathrm{TF}$ \\
\hline Pregrado & $\mathrm{CF}^{\wedge}(\mathrm{SI})^{\wedge} \mathrm{TF}$ \\
\hline Posgrado & $\mathrm{CF}^{\wedge}(\mathrm{CO})^{\wedge}(\mathrm{SI})^{\wedge}(\mathrm{TF})$ \\
\hline Portugués/Brasil & $\mathrm{CF}^{\wedge}(\mathrm{SI})^{\wedge} \mathrm{TF}$ \\
\hline Español/Chile & $\mathrm{CF}^{\wedge}(\mathrm{CO})^{\wedge} \mathrm{SI} \mathrm{T}^{\wedge} \mathrm{TF}$ \\
\hline
\end{tabular}

Al igual que con el Potencial de Estructura Genérica de las introducciones, las conclusiones muestran una fuerte dispersión en los elementos obligatorios y opcionales, lo cual refuerza la noción de clases de textos situados, con variaciones por nivel educativo, lengua y marco institucional, más allá de la disciplina en común. Los elementos comunes al corpus son solo tres: consideraciones finales, síntesis de hallazgos (opcional) y trabajos futuros. Los subcorpus muestran además ciertos elementos característicos: las contribuciones de la tesis son obligatorias en las tesis de posgrado chilenas en español y los elementos iterativos solo aparecen en ese mismo subgrupo, mientras que las publicaciones son un elemento optativo que solo aparece en las tesis de pregrado brasileñas en portugués.

$\mathrm{Al}$ agrupar ejemplares por nivel educativo, el pregrado repite el Potencial de Estructura Genérica del corpus global, mientras que el posgrado gana en complejidad de elementos optativos y obligatorios, incorporando contribuciones (optativo) y reduciendo la obligatoriedad de referirse a trabajos futuros. Al contrastar por lenguas y 
espacios institucionales, los ejemplares en portugués de Brasil repiten el potencial del corpus general, mientras que los ejemplares chilenos en español muestran el Potencial de Estructura Genérica más compleja y obligatoria de todas las agrupaciones: consideraciones finales, contribuciones (optativo), síntesis y trabajos futuros.

\subsection{Opciones de despersonalización}

Por último, se abordó una dimensión de la unidad de textura, realizada mediante opciones léxico-gramaticales, que resulta un desafío importante para la construcción de una voz autoral en los géneros académicos de formación: el uso de mecanismos de personalización (primera persona singular coincidente) y despersonalización dentro del sistema de modo, según puede verificarse en la Tabla 10:

Tabla 1o. Cantidad y porcentaje de opciones de personalización y despersonalización en introducciones y conclusiones de tesis de ingeniería eléctrica por nivel educativo y lengua/institución.

\begin{tabular}{|c|c|c|c|c|c|c|c|c|c|c|c|}
\hline & \multicolumn{2}{|c|}{$\begin{array}{c}\text { Pregrado } \\
\text { Portugués/Brasil }\end{array}$} & \multicolumn{2}{|c|}{$\begin{array}{c}\text { Pregrado } \\
\text { Español/Chile }\end{array}$} & \multicolumn{2}{|c|}{$\begin{array}{c}\text { Posgrado } \\
\text { Portugués/Brasil }\end{array}$} & \multicolumn{2}{|c|}{$\begin{array}{c}\text { Posgrado } \\
\text { Español/Chile }\end{array}$} & \multicolumn{2}{|c|}{ TOTAL } \\
\hline & & $n$. & $\%$ & $n$. & $\%$ & $n$. & $\%$ & $n$. & $\%$ & n. & $\%$ \\
\hline \multirow{3}{*}{$\begin{array}{l}\text { Forma no } \\
\text { finita }\end{array}$} & Int & 7 & 4 & 35 & 13 & 15 & 5 & 38 & 9 & 95 & 8 \\
\hline & Conc & 7 & 4 & 31 & 11 & 9 & 3 & 12 & 3 & 59 & 5 \\
\hline & Total & 14 & 7 & 66 & 24 & 24 & 9 & 50 & 12 & 154 & 13 \\
\hline \multirow{3}{*}{ Pasiva } & Int & 16 & 8 & 22 & 8 & 15 & 5 & 45 & 11 & 98 & 9 \\
\hline & Conc & 8 & 4 & 32 & 12 & 26 & 9 & 52 & 13 & 118 & 10 \\
\hline & Total & 24 & 13 & 54 & 20 & 41 & 15 & 97 & 24 & 216 & 19 \\
\hline \multirow{3}{*}{ Metonimia } & Int & 92 & 48 & 82 & 30 & 98 & 35 & 119 & 30 & 391 & 34 \\
\hline & Conc & 61 & 32 & 73 & 27 & 106 & 38 & 129 & 32 & 369 & 32 \\
\hline & Total & 153 & 80 & 155 & 56 & 204 & 73 & 248 & 62 & 760 & 66 \\
\hline \multirow{3}{*}{$1^{a}$ P. Plural } & Int & 0 & 0 & 0 & 0 & 1 & 0 & 3 & 1 & 4 & 0 \\
\hline & Conc & 0 & 0 & 0 & 0 & 9 & 3 & 1 & 0 & 10 & 1 \\
\hline & Total & 0 & 0 & 0 & 0 & 10 & 4 & 4 & 1 & 14 & 1 \\
\hline \multirow{3}{*}{$\begin{array}{l}1^{\mathrm{a}} \mathrm{P} . \\
\text { Singular }\end{array}$} & Int & 0 & 0 & 0 & 0 & 0 & 0 & 2 & 0 & 2 & 0 \\
\hline & Conc & 0 & 0 & 0 & 0 & 1 & 0 & 0 & 0 & 1 & 0 \\
\hline & Total & 0 & 0 & 0 & 0 & 1 & 0 & 2 & 0 & 3 & 0 \\
\hline \multicolumn{2}{|l|}{ TOTAL } & 191 & 100 & 275 & 100 & 280 & 100 & 401 & 100 & 1147 & 100 \\
\hline
\end{tabular}

Los resultados muestran la baja incidencia de la primera persona singular coincidente (con apenas 3 ocurrencias en dos ejemplares, ambos del posgrado) y la primera persona plural (14 ocurrencias en cinco ejemplares, también exclusivamente del posgrado) en relación con el total de ocurrencias de opciones de personalización y despersonalización (1147 ocurrencias en el corpus completo). Esto significaría que la manifestación de un lugar de enunciación más personalizado es infrecuente en la disciplina, más allá de la lengua e institución, y las escasas apariciones requieren un escritor en un lugar de experticia mayor (posgrado). Dentro de las formas despersonalizadas, predomina la metonimia (66\%), mientras que las formas pasivas $(19 \%)$ y no finitas $(13 \%)$ son mucho menos frecuentes. A continuación, se ofrecen ejemplos de estos tres tipos de opciones, con los recursos léxico-gramaticales subrayados: 
Tabla 11. Opciones más frecuentes de despersonalización en tesis de ingeniería eléctrica.

\begin{tabular}{|c|c|c|}
\hline & Portugués/Brasil & Español/Chile \\
\hline $\begin{array}{l}\text { Forma } \\
\text { no } \\
\text { finita }\end{array}$ & $\begin{array}{l}\text { Importante ressaltar que grande parcela da tarifa } \\
\text { de eletricidade no Brasil deve-se à enorme } \\
\text { quantidade de encargos e à elevada carga } \\
\text { tributária incidente, que correspondem, em } \\
\text { média, a } 31,2 \% \text { da tarifa industrial, conforme } \\
\text { ilustra a tabela I.2 (ID15, introducción tesis } \\
\text { doctorado). }\end{array}$ & $\begin{array}{l}\text { Además, considerar la variación de } \\
\text { velocidad en la formulación de los } \\
\text { controladores difusos y controlador } \\
\text { predictivo híbrido, trabajo ya adelantado } \\
\text { en las secciones } 5.4 .4,6.2 .5 \text { y } 7.3 .3 \text { (ID } 04 \text {, } \\
\text { conclusión tesis doctorado). }\end{array}$ \\
\hline Pasiva & $\begin{array}{l}\text { Quanto ao desenvolvimento de projetos com } \\
\text { computadores de baixo custo como a Raspberry } \\
\mathrm{Pi} \text {, nota-se o constante crescimento do número } \\
\text { de projetos e aplicações muito em virtude da } \\
\text { comunidade de desenvolvedores espalhada pelo } \\
\text { mundo que compartilham muitas informações, } \\
\text { e também o alto número de softwares de código } \\
\text { aberto (ID16, conclusión tesis pregrado). }\end{array}$ & $\begin{array}{l}\text { Se realiza una revisión de la normativa } \\
\text { local, normativa internacional y } \\
\text { recomendaciones internacionales sobre } \\
\text { emisiones electromagnéticas para sistemas } \\
\text { celulares (ID } 07 \text { introducción tesis } \\
\text { pregrado). }\end{array}$ \\
\hline $\begin{array}{l}\text { Meto- } \\
\text { nimia }\end{array}$ & $\begin{array}{l}\text { Este projeto contemplou o uso e } \\
\text { intercomunicação de diferentes linguagens de } \\
\text { programação como Python, scripts de } \\
\text { inicialização e comandos Linux, linguagem de } \\
\text { programação dedicada a Arduino, e } \\
\text { intercomunicação entre os programas Arduino e } \\
\text { Linux (ID17, conclusión tesis pregrado). }\end{array}$ & $\begin{array}{l}\text { Con respecto a los métodos de } \\
\text { reconocimiento, el estudio comparativo } \\
\text { muestra que los métodos basados en } \\
\text { características LBP son más robustos ante } \\
\text { rotaciones de los rostros que los métodos } \\
\text { Gabor y WLD en ambientes indoor } \\
\text { (ID02, conclusión tesis doctorado). }\end{array}$ \\
\hline
\end{tabular}

$\mathrm{Al}$ distinguir por nivel educativo y lengua/contexto institucional, se verifica que la metonimia es más frecuente en las tesis brasileñas en portugués (promedio: 77\%) que en las tesis chilenas en español (promedio: 59\%); por el contrario, las formas de la voz pasiva son más frecuentes en español en Chile (promedio: 22\%) que en portugués en Brasil (promedio: 14\%), y las formas no finitas se destacan por su presencia en las tesis de pregrado en español de Chile (24\%). Por último, el contraste entre secciones de la estructura de las tesis muestra una llamativa consistencia y balance: la distribución de las ocurrencias de formas de personalizar y despersonalizar la voz autoral es casi idéntica en introducciones y conclusiones.

\section{CONCLUSIONES}

En esta investigación se buscó describir algunos rasgos contextuales, estructurales y discursivos del género discursivo tesis en ingeniería eléctrica contrastando el nivel educativo (pregrado y doctorado) y la lengua (español y portugués) en dos universidades estatales metropolitanas tradicionales y altamente selectivas de Chile y Brasil. Para tal fin, se utilizó el marco teórico de la Lingüística Sistémico-Funcional y el concepto de Potencial de Estructura Genérica, especialmente relevante para comprender cómo las opciones lingüísticas disponibles a los hablantes y escritores se tipifican en potenciales semióticos, semánticos y léxico-gramaticales. Se analizó cualitativamente la Configuración Contextual, la extensión, el Potencial de Estructura Genérica de elementos iniciales, y el Potencial de Estructura Genérica de elementos 
semánticos y las opciones de despersonalización en la introducción y la conclusión, dos secciones de fuerte construcción retórica.

El género tesis se inscribe en una Configuración Contextual de asimetría de experticia y saber entre autor y director, roles que en ingeniería eléctrica son predominantemente ocupados por hombres, y varía en su complejidad y aporte al saber consensuado experto según el nivel educativo. Consiste en una investigación individual sistemática sobre un tema especializado, en un marco de educación formal superior, y tiene como producto un texto escrito multimodal. Su extensión ronda las 96 páginas en pregrado y el doble de extensión en posgrado, aunque en español en Chile el contraste entre niveles educativos es mayor. Las tesis se inician obligatoriamente con tapa, dedicatoria, agradecimientos y resumen en lengua local, elementos vinculados a la inscripción institucional y la indexación, pero también a dimensiones interpersonales de vínculo con el entorno personal y profesional; las tesis brasileñas incluyen muchos más elementos obligatorios relacionados con la inclusión de componentes multimodales y con la protocolarización del producto, factor que puede estar vinculado con tendencias locales de acreditación e internacionalización de la educación superior. A continuación, las introducciones incluyen obligatoriamente la motivación y objetivos de la investigación, junto con su organización textual; las tesis de posgrado exhiben un Potencial de Estructura Genérica más complejo y estable, con elementos optativos como la fundamentación general, la fundamentación teórica y la metodología. En las conclusiones, resultan elementos obligatorios las consideraciones finales y los trabajos futuros, y optativa la síntesis de hallazgos; el posgrado gana en complejidad de elementos optativos y obligatorios, incorporando contribuciones (optativo) y reduciendo la obligatoriedad de referirse a trabajos futuros, mientras que, al agrupar por lenguas, las tesis en español muestran una estructura potencial más sofisticada. Los elementos iterativos, otro rasgo de complejidad escritural, son propios de algunos elementos en el nivel de posgrado. Esta diversidad de rasgos del género según las variables consideradas, incluso dentro de cada subcorpus, refuerza la necesidad de continuar realizando investigaciones de clases de textos situados, en particular si se requiere contar con evidencias para fundamentar iniciativas locales de enseñanza y apoyo en el proceso de escritura de textos. La presencia textual del escritor, en la primera persona gramatical singular coincidente o en la primera persona de autor en plural, es prácticamente inexistente en introducciones y conclusiones, que optan por estrategias y recursos léxico-gramaticales de despersonalización, especialmente la metonimia. Esta elección textual puede vincularse con un marco epistemológico de la disciplina en el que la subjetividad y el posicionamiento del autor deben camuflarse y disimularse.

Los aspectos relevados no solo son centrales para el conocimiento del género, sino que también constituyen desafíos posiblemente novedosos para los escritores en formación. En consecuencia, los resultados hallados en esta investigación pueden 
orientar las iniciativas de alfabetización disciplinar destinadas a estudiantes de ingeniería de pregrado y posgrado en Chile y Brasil, e incluso servir como evidencias para confirmar, comparar o descartar en otros contextos geográficos e institucionales de la región.

Cabe aclarar que los resultados de esta investigación se extrajeron de un corpus limitado y se circunscribieron a dos universidades; por tanto, no deben ser extrapolados sin más a otras instituciones o países. Sin embargo, la tradición e influencia de las instituciones investigadas en sus respectivos contextos, y el alto número relativo de ocurrencias de los fenómenos analizados, permite concluir que sugieren patrones propios de la disciplina, las lenguas y los niveles educativos abordados. Futuras investigaciones deberán confirmar, relativizar o rechazar las conclusiones aportadas.

\section{REFERENCIAS BIBLIOGRÁFICAS}

Agüero, C. (2014). ¿Conforman las sentencias penales un género discursivo? Estudios Filológicos, 53, 7-26.

Araújo, A. D. (2006). Práticas discursivas em conclusões de teses de doutorado. Linguagem em (Dis)curso, 6(3), 447-462.

Arnoux, E. (Ed.) (2009). Escritura y producción de conocimiento en las carreras de posgrado. Buenos Aires: Santiago Arcos Editor.

Bonilla Dávila, N. A. (2016). Experiencia académica de estudiantes mujeres en primer año de ingeniería. Propuestas de mejoramiento de los sistemas de apoyo a estudiantes con mirada de género. Memoria inédita de pregrado, Universidad de Chile, Chile.

Bowcher, W. L. \& Yameng Liang, J. (2016). GSP and multimodal texts. En W. L. Bowcher \& J. Yameng Liang (Eds.), Society in Language, Language in Society. Essays in Honour of Ruqaiya Hasan (pp. 251-274). Hampshire: Palgrave MacMillan.

Caffarella, R. \& Barnett, B. (2000). Teaching Doctoral students to become scholarly writers: The importance of giving and receiving critiques. Studies in Higher Education, 25(1), 39-52.

Carlino, P. (2013). Alfabetización académica diez años después. Revista Mexicana de Investigación Educativa, 18(57), 355-381.

Castro Azuara, M. C. \& Sánchez Camargo, M. (2015). Escribir en la universidad: La organización retórica del género tesina en el área de humanidades. Perfiles Educativos, XXXVII(148), 50-67. 
Chiroleu, A. \& Marquina, M. (2017). Democratisation or credentialism? Public policies of expansion of higher education in Latin America. Policy Reviews in Higher Education, 1(2), 139-160.

Connor, U. \& Moreno, A. I. (2005). Tertium comparationis. A vital component in contrastive rhetoric research. En P. Bruthiaux, D. Atkinson, W. G. Eggington, W. Grabe \& V. Ramanathan (Eds.), Directions in Applied Linguistics. Essays in Honor of Robert B. Kaplan (pp. 153-164). Clevedon, Buffalo, Toronto: Multilingual Matters Ltd.

Controladoria Geral (2016). Anuário estatístico. São Paulo: USP.

Eggins, S. (2004). An introduction to systemic functional linguistics. Londres: Pinter.

Eodice, M., Geller, A. E. \& Lerner, N. (2016). The meaningful writing project. Learning, teaching, and writing in higher education. Boulder, CO: Utah State University Press.

Feak, C. B. \& Swales, J. M. (2011). Creating contexts: Writing introductions across genres. Ann Arbor, MI: University of Michigan Press.

García Negroni, M. M. (2008). Subjetividad y discurso científico-académico. Acerca de algunas manifestaciones de la subjetividad en el artículo de investigación en español. Revista Signos. Estudios de Lingüística, 41(66), 5-31.

Gardner, S. \& Nesi, H. (2013). A classification of genre families in university student writing. Applied Linguistics, 34(1), 25-52.

Halliday, M. A. K. \& Matthiessen, C. M. I. M. (2014). An introduction to functional grammar. Londres \& Nueva York: Routledge.

Hasan, R. (1989). The structure of a text; the texture of a text; the identity of a text. En M. A. K. Halliday \& R. Hasan (Eds.), Language, context and text: aspects of language in a social-semiotic perspective (pp. 55-118). Oxford: Oxford University Press.

Hasan, R. (1996). The nursery tale as genre. En C. Cloran, D. Butt \& G. Williams (Eds.), Ways of Saying: Ways of Meaning. Selected Papers of Ruqaiya Hasan (pp. 5172). Londres \& Nueva York: Cassell.

Hill, C., Corbett, C. \& Rose, A. S. (2010). Why so few? Women in Science, Technology, Engineering, and Mathematics. Washington DC: American Association of University Women.

Ibáñez, R. (2008). El texto disciplinar y el acceso al conocimiento desde el análisis del género: ¿Regulación del conocimiento o persuasión? En G. Parodi (Ed.), Géneros académicos y géneros profesionales: Accesos discursivos para saber y hacer (pp. 219-246). Valparaíso: Ediciones Universitarias de Valparaíso. 
Kanoksilapatham, B. (2007). Rhetorical moves in biochemistry research articles. En D. Biber, U. Connor \& T. A. Upton (Eds.), Discourse on the move. Using corpus analysis to describe discourse structure (pp. 73-119). Ámsterdam \& Filadelfia: John Benjamins.

Lim, J. M.-H. (2014). Formulating research questions in experimental doctoral dissertations on Applied Linguistics. English for Specific Purposes, 35, 66-88.

Martínez Hincapié, J. D. (2015). El género Tesis Doctoral de Historia y Física: Descripción y variación retórico-funcional. En G. Parodi \& G. Burdiles (Eds.), Leer y escribir en contextos académicos y profesionales: Géneros, corpus y métodos (pp.113-152). Santiago de Chile: Ariel.

Medeiros, R. (2015). Resumo de dissertações e teses: A estrutura composicional à luz da Análise Textual dos Discursos. Tesis doctoral inédita, Universidade Federal do Rio Grande do Norte, Natal, Brasil.

Meza, P. \& Sabaj, O. (2016). Funciones discursivas de consenso y disenso en tesis de lingüística. Onomázęin, 33, 385-411.

Navarro, F. (2015). Análisis situado del plan de negocios en español y portugués: Perspectivas de emprendedores, docentes y estudiantes. Calidoscópio, 13(2), 189-200.

Navarro, F. (2018). Más allá de la alfabetización académica: Las funciones de la escritura en educación superior. En M. A. Alves \& V. Iensen Bortoluzzi (Eds.), Formação de Professores: Ensino, linguagens e tecnologias (pp. 13-49). Porto Alegre: Editora Fi.

Oyebode, O. \& Unuabonah, F. O. (2013). Coping with HIV/AIDS: A multimodal discourse analysis of selected HIV/AIDS posters in south-western Nigeria. Discourse \& Society, 24(6), 810-827.

Paré, A., Starke-Meyerring, D. \& McAlpine, L. (2011). Knowledge and identity work in the supervision of Doctoral student writing: Shaping rhetorical subjects. En D. Starke-Meyerring, A. Paré, N. Artemeva, M. Horne \& L. Yousoubova (Eds.), Writing in Knowledge Societies (pp. 215-236). Fort Collins, Colorado: The WAC Clearinghouse \& Parlor Press.

Parodi, G. (2008). El género Manual y su organización retórica en cuatro disciplinas científicas: Entre la abstracción y la concreción. En G. Parodi (Ed.), Géneros académicos y géneros profesionales: Accesos discursivos para saber y hacer (pp. 199-218). Valparaíso: Ediciones Universitarias de Valparaíso. 
Pereira, C. C. (2012). A produção do gênero monografia em discursos de professores e alunos do curso de letras. Tesis doctoral inédita, Universidade Federal do Rio Grande do Norte, Natal, Brasil.

Russell, D. (2013). Contradictions regarding teaching and writing (or writing to learn) in the disciplines: What we have learned in the USA. Revista de Docencia Universitaria, 11(1), 161-181.

Savio, A. K. (2015). Desobedeciendo el ideal: La producción de tesis en psicoanálisis. Traslaciones, 2(3), 99-126.

Simões, A. C. (2016). El estado de arte de la teoría de Ruqaiya Hasan: ¿Dónde están las pesquisas sobre el Potencial de Estructura Genérica (PEG)? Texturas, 15, 83-96.

Solar, I. J. (2013). Descripción funcional de introducciones de tesis doctorales en las disciplinas de química y lingüística. Onomázein, 28, 72-87.

Swales, J. M. (2004). Research genres. Exploration and applications. Glasgow: Cambridge University Press.

Tapia Ladino, M. \& Burdiles, G. (2012). La organización retórica del marco referencial en tesis de trabajo social. Alpha, 35, 169-184.

Thaiss, C. J. \& Zawacki, T. M. (2006). Engaged writers and dynamic disciplines: Research on the Academic Writing Life. Portsmouth, NH: Boynton/Cook.

Venegas, R., Núñez, M. T., Zamora, S. \& Santana, A. (2015). Escribir desde la Pedagogía del Género. Guías para escribir el Trabajo Final de Grado en Licenciatura. Valparaíso: PUCV.

Xu, X., Wang, Y., Forey, G. \& Li, L. (2010). Analyzing the genre structure of Chinese call-center communication. Journal of Business and Technical Communication, 24(4), 445-475.

Yuen, C. Y. (2004). The construal of ideational meaning in print advertisements. En K. O'Halloran (Ed.), Multimodal discourse analysis. Systemic Functional Perspectives (pp. 163-195). Londres \& Nueva York: Continuum.

\section{AGRADECIMIENTOS}

* Se agradece el financiamiento otorgado por la bolsa de doutorado sanduíche de la Coordenação de Aperfeiçoamento de Pessoal de Nivel Superior (CAPES/PDSE 88881.134065/2016-01, Brasil), el Fondo Basal para Centros de Excelencia proyecto FB0003 de PIA-CONICYT, el Proyecto FONDECYT de iniciación No 11160856 de CONICYT y el Plan de Mejoramiento Institucional (PMI) UCH1501 del Ministerio de 
Educación de Chile, que permitieron la realización de esta investigación. Además, se agradecen las valiosas sugerencias de Annabelle Lukin (Macquarie University), de los evaluadores pares anónimos y de los editores de la Revista Signos. Estudios de Lingüistica sobre versiones preliminares de este artículo. 